\title{
Portable device for thermal nondestructive testing of hidden corrosion in metallic shells by using a LED heat source
}

\author{
D.A. Simonov ${ }^{*}$, A.I. Moskovchenko ${ }^{1}$ \\ ${ }^{1}$ Tomsk Polytechnic University, Tomsk, Russia
}

\begin{abstract}
Thermal nondestructive testing (NDT) is a promising technique for detecting hidden corrosion in metallic shells, such as above-ground tanks, pipes, containers, etc., due to its high productivity and illustrativeness. However, the use of powerful halogen lamps in practical applications is questionable because of reflected radiation and safety requirements. In this paper, a portable thermal NDT device using a LED heater is described. Such heaters operate at wavelengths which are beyond of the spectral band of contemporary infrared imagers. Also, they can use a battery as a power supply thus meeting in-field requirements, for example, in the petrochemical industry. Depending on defect size and material loss, the developed portable thermal NDT device can detect corrosion in steel shells with thickness up to $8 \mathrm{~mm}$.
\end{abstract}

\section{Introduction}

Every year, the degradation of metallic parts and constructions causes huge economical and energy losses related to restoration and liquidation of consequences of accidents and catastrophes in nuclear engineering, power production, petrochemical and boat production industries, etc. The detection of corrosion in metallic shells of different size and mass is an important problem of condition monitoring and predictive maintenance. The conventional nondestructive testing (NDT) methods used in corrosion detection are, first of all, ultrasonics and $\mathrm{X}$ ray $[1,2]$. However, the both techniques are timeconsuming thus preventing their use in testing of large areas subjected to obligatory inspection.

One of the most promising express inspection techniques is thermal testing (TT), of which scheme is presented in Figure 1. A classical TT procedure implements thermal stimulation of a test object by means of an optical heat source (convective and inductive heat sources are also used) and the parallel monitoring of a test object surface with an infrared (IR) imager. A sequence of IR images called "thermograms" is recorded on a computer to be further analysed by using a couple of data processing techniques, such as the Fourier and wavelet transforms, principal component analysis, nonlinear fitting, etc. Test results are presented in the form of binary maps of defects. In the case of large area to be inspected, a map of defects can be obtained by combining IR thermograms in a single resulting image.

The first applications of active TT were in the aviation industry. In 1994, Prabhu and Winfree used a neural network to enhance detectivity of hidden corrosion in airframes [3]. The research in this area was summarized by Alcott [4]. In the next years, potentials of TT were extended onto inspection of steel parts [5, 6], and this application area was recently reviewed in [7].

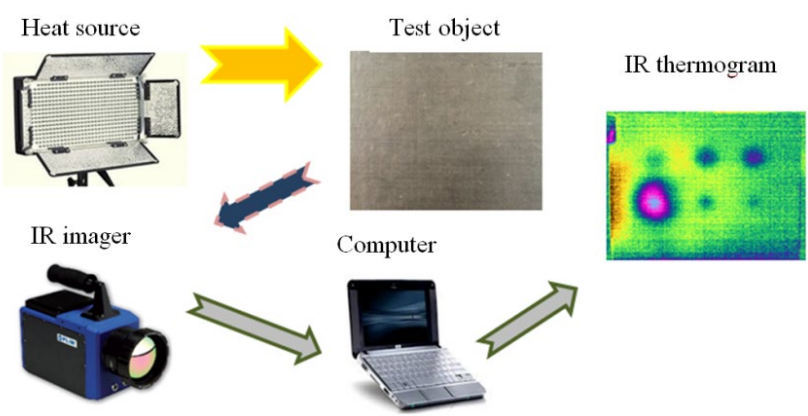

Fig. 1. Scheme of active one-sided thermal nondestructive testing.

\section{Practical significance}

Since years, the Thermal Testing laboratory at National Research Tomsk Polytechnic University has been developing the method and apparatus for TT of nonmetallic and metallic materials with the emphasis being done on the evaluation of composites widely used in the aerospace industry [8-9]. In the field of the detection of corrosion in metallic parts with thickness of up to 10 $\mathrm{mm}$, it has been demonstrated that TT is capable to detect material loss of about $10 \%$ [10-17]. However, it is worth stressing that, in general, the inspection of metals by using TT is more difficult in comparison to TT of

\footnotetext{
* Corresponding author: $\underline{\mathrm{s} \text { disa } @ \text { mail.ru }}$
} 
non-metals. Metals are characterized by lower than nonmetals absorption toward heating radiation and higher thermal diffusivity. As a result, in metals, temperature signals in defect areas are low and exist for short times. The research fulfilled at the Thermal Testing laboratory has shown that in TT of $1 \mathrm{~mm}$-thick steel containers intended for keeping radioactive wastes one should apply Xenon flash tubes as a powerful and pulsed (flash duration about $5 \mathrm{~ms}$ ) heat source (Figure 2a).

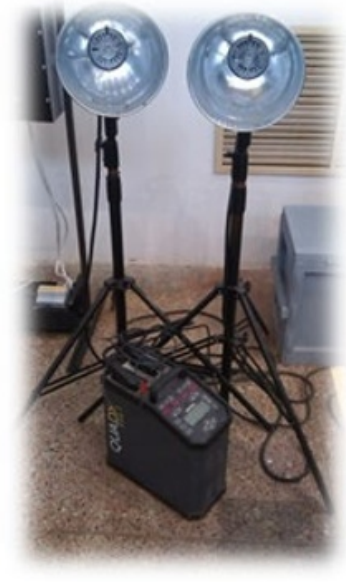

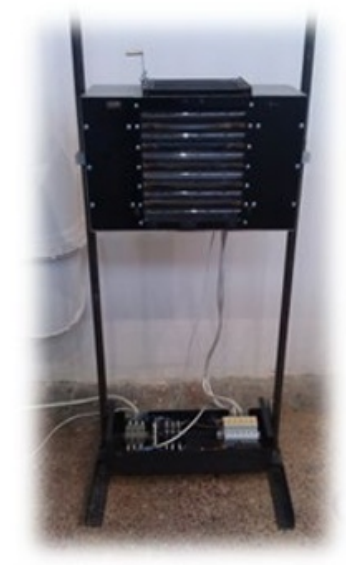

b
Fig. 2. Heat sources used in thermal testing: a - Xenon flash tubes, energy $3.2 \mathrm{~kJ}, \mathrm{~b}$ - halogen lamps, power $30 \mathrm{~kW}$.

However, in the case of thick metals, it is difficult to pump enough energy in a bulk material with Xenon tubes, therefore, powerful halogen lamps (Figure 2b) are an alternative heat source of whose power may reach 30 $\mathrm{kW}$. A disadvantage of using halogen lamps in TT is the presence of reflected spurious radiation which appears at both the heating and cooling stages (in the latter case, it is a residual radiation of lamp balloons). In order to subdue specific thermal noise, some special data processing algorithms should be used in TT typically at a cooling stage, but, at longer observation times, heat diffusion may seriously "smash" thermal patterns of hidden defects.

\subsection{Choosing a heat source}

With the above-mentioned taken into account, it may be concluded that the use of energy-consuming Xenon and halogen lamps in active TT is difficult in field conditions where rigid safety requirements, for example, taking place at petrochemical plants, often prevent implementation of high-voltage $(220 \mathrm{~V})$ equipment. The fast development of micro- and nano-electronics has stimulated the appearance of both miniature IR cameras and compact Laser Emitting Diodes (LED). At present, powerful LEDs are considered as candidate heat sources in active TT. A remarkable feature of such light emitters is that they cannot "blind" sensitive IR imagers because they operate at wavelengths less than $1 \mu \mathrm{m}$, i.e. far away from the typical wavelength ranges of contemporary IR imagers, namely, 3-5 and 7-14 $\mu \mathrm{m}$.

\section{Creating a portable flaw detector}

This paper describes the results of development of a portable powerful LED source as a major component of the thermal NDT device intended for non-contact detection of corrosion in metallic shells (Figure 3 ).

It is believed that such TT device can be used to detect hidden corrosion in large-size metallic parts and installations widely used in the modern industry, in particular, at petrochemical plants.
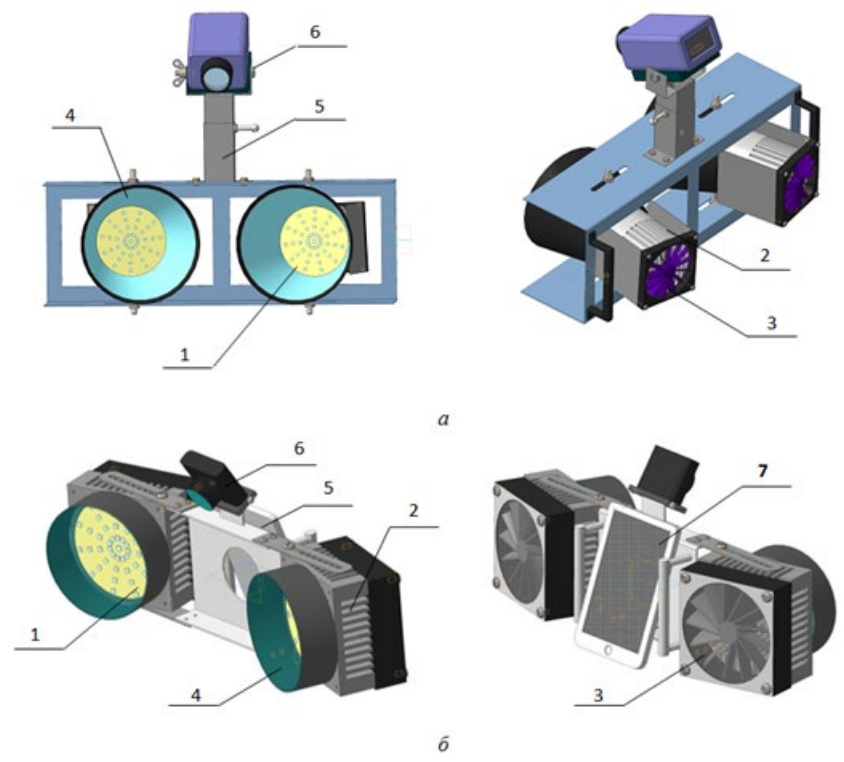

Fig. 3. Thermal NDT equipment: 1 - LED panels (total power $1 \mathrm{~kW}), 2$ - aluminium heat sink radiators, 3 - electric ventilators, 4 - light-reflecting trumpets, 5 - fasteners, 6 - IR thermographic module, 7 - tablet computer.

\subsection{Scientific novelty}

A novelty of this work is in the use of LEDs as a heat source in active TT that is characterized by a number of advantages, namely: 1) a low level of thermal reflections produced by LED panels, mainly due to a lower temperature of the panels compared to halogen lamps, 2) a narrow spectral band of LED radiation that is out of operation spectral ranges of most IR imagers; this fact excludes reflected noisy signals during heating, 3) low operation voltage of LED panels and the possibility to use batteries as power supply sources for TT devices during one work shift. However, it is worth indicating that one of a major disadvantages of using LED sources for heating is a high level of reflection of LED radiation from many materials, in particular, those painted in light colours; this might significantly diminish absorbed heating energy and, hence, reduce surface temperature of test objects thus causing, in its turn, weaker temperature signals in defect areas. 


\subsection{Application area}

The main application area for the developed TT device is the evaluation of corrosion material loss in steel tanks, containers and piping having thickness of less than $8 \mathrm{~mm}$ and a curvature radius less than $0.4 \mathrm{~m}$. Such metallic shells are ubiquitous in the petrochemical and power production industries being used for keeping and transportation of gas and oil products and low-activity radioactive wastes.

The economic efficiency of using a developed noncontact inspection device in NDT of metallic parts is a considerable saving of time and labour-cost to compare to a conventional technique of point-by-point ultrasonic thickness gauging.

\section{Description of the experiment}

A series of experiments on corrosion detection has been fulfilled at the Thermal Testing laboratory on a special reference sample. The sample represented a cube made of steel plates with thicknesses of 2, 4, 6 and $8 \mathrm{~mm}$ and filled with water. The rear cube surface contained 30 sites of material loss having different shape and size. An example of the location of defects is shown in Figure 4a, the reference sample photo is presented in Figure $4 \mathrm{~b}$.

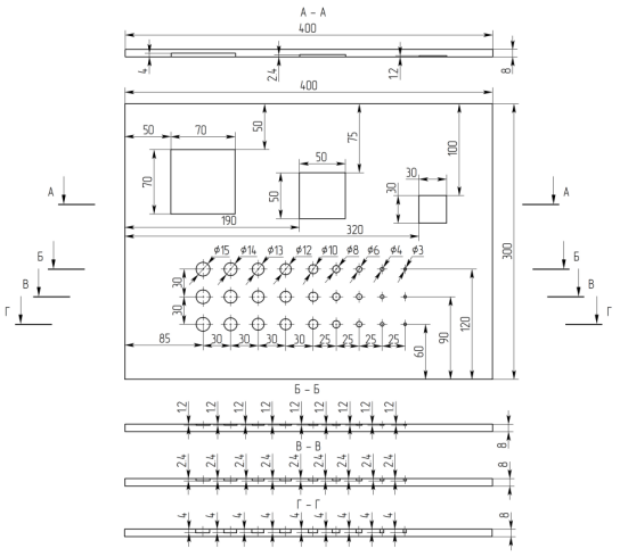

a

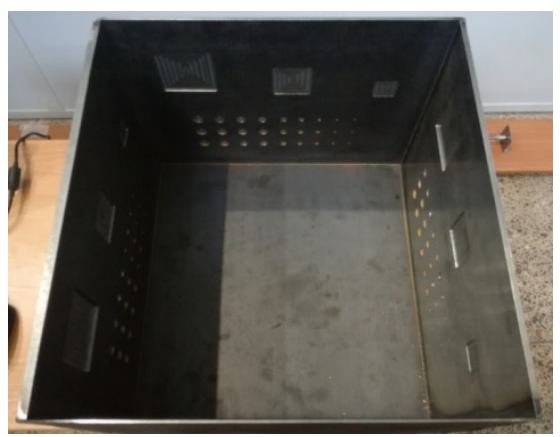

b

Fig. 4. Reference sample with artificial defects simulating rearsurface corrosion: a - scheme of defects ( $8 \mathrm{~mm}$-thick wall), $\mathrm{b}$ - reference sample photo.
The test procedure by using a powerful LED heat source is illustrated by Figure 5 .

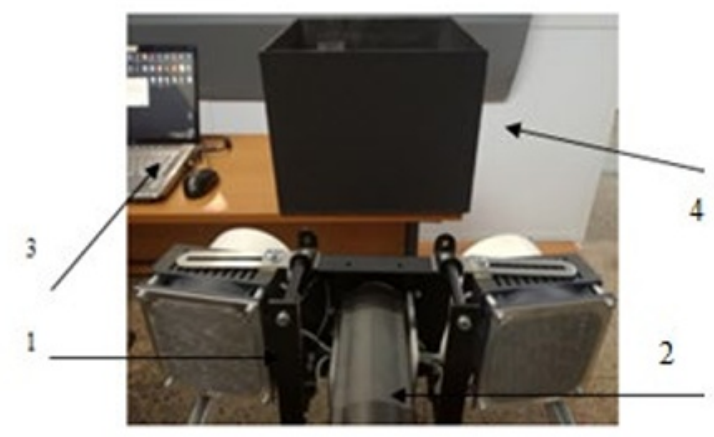

Fig. 5. Active TT procedure: 1- powerful LED source, 2 - IR imager, 3- computer, 4- reference sample.

The operation of the developed LED heat source is illustrated by Figure 6; note that the reference sample (Figure 4) was black-painted to improve absorption of heating radiation.

The distance between the tested surface and the LED heater equal to $0.4 \mathrm{~m}$ was optimized to ensure a reasonable excess temperature of the reference sample and a larger inspected area $(240 \times 180 \mathrm{~mm})$.

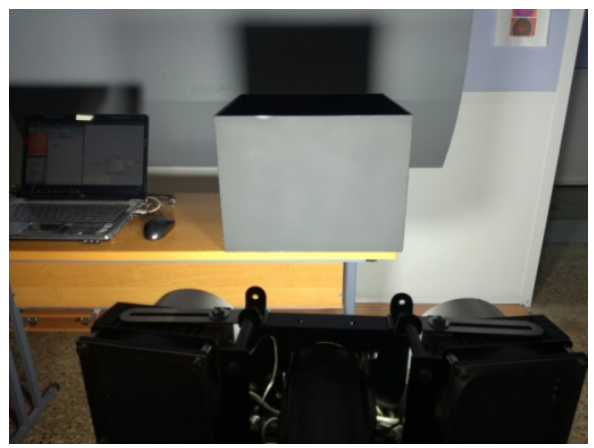

Fig. 6. Powerful LED heat source in operation.

In order to thermographically cover the heated area, the IR imager has been placed at the distance of $1.1 \mathrm{~m}$ from the sample.

The temperature distribution on the surface of the reference sample was monitored by means of a FLIR A325sc IR thermographic module of which technical characteristics are presented in Table 1.

Table 1. Technical performance of FLIR A325sc IR thermographic module.

\begin{tabular}{|l|l|}
\hline Detector & Microbolometric array \\
\hline Detector array size, pixel & $320 \times 240$ \\
\hline Temperature sensitivity, 0C & 0.06 \\
\hline Frame frequency, Hz & up to 60 \\
\hline
\end{tabular}


Table 2. Results of active TT in application to detection of hidden corrosion in reference sample.

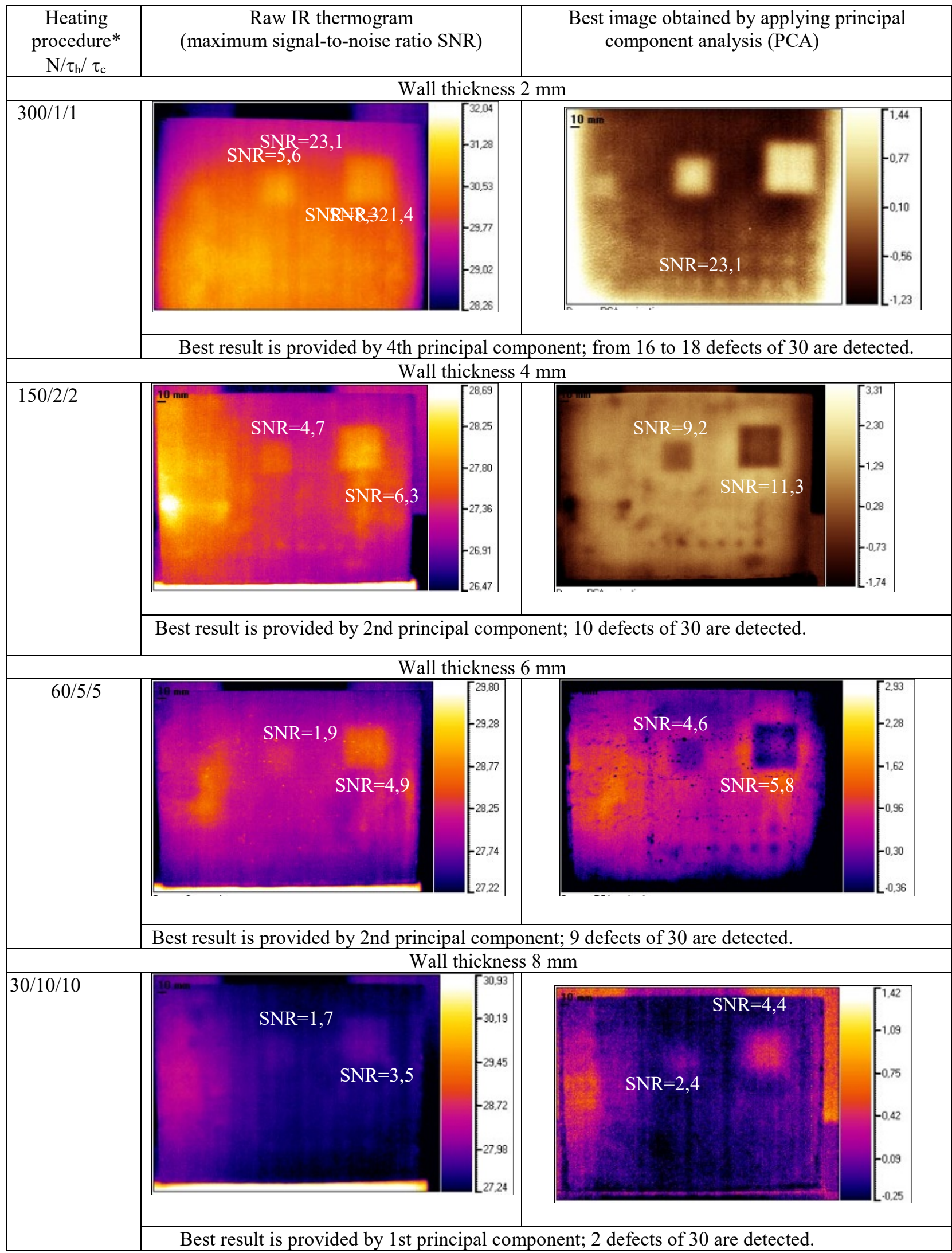

*Number of heating cycles/Heating duration, s/Cooling duration, s. 


\section{Results}

Some results of corrosion detection are presented in Table 2. A thermal wave test procedure implementing cyclic heating has been used in the experiments. Heating parameters have been optimized by using a numerical analysis of test cases performed by means of a ThermoCalc-3D program developed at Tomsk Polytechnic University for modelling 3D TT problems.

Signal-to-noise ratio (SNR) has been chosen as a test efficiency criterion; it has been determined over the defects with the largest area. Besides, the test efficiency has been also evaluated by the number of the defects detected on each sample wall.

Inspection results have been significantly affected by the presence of water in the reference cube in the case of the $2 \mathrm{~mm}$-thick wall. If there was no water in the cube, 17 defects were detected, while in the filled with water cube the number of detected defects reduced up to 12 . Also, the values of signal-to-noise ratio were about twice higher in the case of the empty reference cube. Similar results were observed when inspecting the 4 and 6-mm thick cube walls. Surprisingly, the test results improved in the case of defects in the $8 \mathrm{~mm}$-thick wall: 2 detected defects in the empty cube against 8 defects in the filled with water sample (signal-to-noise ratio remained about the same).

\section{Conclusion}

In the framework of this project, a portable powerful LED heater was developed as an important component of the TT device. Such heat source enables the detection of material loss in steel shells with thickness up to $6 \mathrm{~mm}$ (in some cases $-8 \mathrm{~mm}$ ) with the test productivity of about $5 \mathrm{~m}^{2} / \mathrm{hr}$. The heater can be combined with a couple of commercial IR imagers, such as FLIR A325, OPTRIS PI460, ThermApp, and tablet computers. The use of LED panels with the total power of $1 \mathrm{~kW}$ operating at wavelengths $(0.4-0.9 \mu \mathrm{m})$ beyond a range of spectral sensitivity of contemporary IR imagers has allowed reducing additive reflection noise by about $50 \%$ to compare to halogen lamps. Altogether, the results obtained allow the development of portable thermal NDT devices oriented toward detection of various types of defects in metallic parts and installations.

\section{References}

1. Ultrasonic inspection of walls and welds while utilizing and repairing vertical steel tanks. -
Directive document RD 19.100.00-KTP-545-06, Russia, 54 (in Russian)

2. Guidelines on supervision for construction and assembly works performed on nuclear-and radiation-hazardous objects. - Directive document RD 03-33-95, Russia, 86 (in Russian)

3. D.R. Prabhu, W.P. Winfree. Proceedings of "Rev. Progress in Quant. NDE" Conf. 12 (1993)

4. J. Alcott. Materials Evaluation 5 (1994)

5. V.P. Vavilov, A.O. Chulkov Proc. SPIE "Thermosense-XXXIV" 8354, 117 (2012)

6. G. Cadelano, A. Bortolin, G. Ferrarini, B.M. Agnellini, D. Giantin, P.P. Zonta, P. Bison. Journal of Nondestructive Evaluation 35 (3) (2016)

7. S. Doshvarpassand, C. Wu, X. Wang. Infrared Physics and Technology 96 (2019)

8. V.P. Vavilov, Composites Part B: Engineering 6, 1 (2014)

9. V.P. Vavilov, D.A. Nesteruk, A.B. Plesovskikh, A.O. Chulkov, Composites: Part B: Engineering 68, 375 (2015)

10. V.P. Vavilov, A.O. Chulkov, Proceedings of 11 th European Conference on Non-Destructive Testing., Brno, 1 (2014)

11. S. Sojasi, X. Maldague, V.P. Vavilov, A.O. Chulkov, et.al. Proceedings of NDT in Canada 2015 Conference, 8 (2015)

12. V.P. Vavilov, A.O. Chulkov, D.A. Derusova Proceedings of the 10th International conference on applied and theoretical mechanics 14, 206 (2014)

13. V.P. Vavilov, A.O. Chulkov, D.A. Derusova Proceedings of SPIE "Thermosense-XXXVI" 9105 (2014)

14. V.P. Vavilov, V.V. Shiryaev, A.O. Chulkov Proceedings of SPIE - The International Society for Optical Engineering 9485 (2015)

15. A.O. Chulkov, V.P. Vavilov Journal of Physics: Conference Series, 671 (2016)

16. A.O. Chulkov, V.P. Vavilov, A.S. Malakhov Russian Journal of Nondestructive Testing 52, 588 (2016)

17. A.O. Chulkov, V.P. Vavilov IOP Conf. Series: Materials Science and Engineering 81, 1 (2015) 